\title{
Synthesis of tetra- and pentacyclic carbazole-fused imides as potential antitumor agents
}

\author{
Norbert Haider,* Rami Jbara, Johann Käferböck, and Ursula Traar \\ Department of Drug and Natural Product Synthesis, Faculty of Life Sciences, \\ University of Vienna, Althanstraße 14, A-1090 Vienna, Austria \\ E-mail: norbert.haider@univie.ac.at
}

Dedicated to Professor Henk C. van der Plas on the occasion of his $80^{\text {th }}$ birthday

\begin{abstract}
A series of tetra- and pentacyclic imides with a carbazole skeleton and a basic side chain at the imide nitrogen was synthesized by cyclization of carbazole-2,3-dicarboxylic acid esters with an appropriate amine or via an $\mathrm{N}$-aminoimide as a reactive intermediate. The target compounds 6 and 9 were tested in vitro for tumor cell-growth inhibition
\end{abstract}

Keywords: Carbazole, pyrrolo[3,4- $b]$ carbazole, imides, antitumor activity

\section{Introduction}

Based on the planar, aromatic tetracyclic skeleton of the antitumor alkaloids, ellipticine and olivacine, ${ }^{1}$ a number of drug candidates with enhanced antineoplastic properties has been developed in the past decades. ${ }^{2}$ Examples include compounds like retelliptine, ${ }^{3}$ pazelliptine, ${ }^{4}$ datelliptium, ${ }^{5}$ or S16020-2. ${ }^{6}$ It has been shown that the presence of a basic side chain of the $N, N$ dialkylaminoalkyl type enhances the drugs' DNA affinity. ${ }^{3}$ The latter is essential for the biochemical mode of action, through stabilisation of the complex formed between DNA and the enzyme, topoisomerase II. ${ }^{7}$ Among the numerous drugs targeting topoisomerase II, also a group of compounds featuring a naphthalene (or higher annulated) ring system fused to a sixmembered cyclic imide structure with a basic $N$-substituent has received considerable attention. The drug molecules, amonafide ${ }^{8}$ and azonafide ${ }^{9}$ can be regarded as prototypes for this type of agent. Remarkably, amonafide as well as the pyrido[4,3-b]carbazole derivative, S16020-2 have been found to escape the P-glycoprotein-mediated multi-drug resistance, ${ }^{10,11}$ the latter being a common problem in tumor chemotherapy. As it has been claimed that certain carbazoles fused to a $N$-substituted cyclic imide also exhibit pronounced antitumor properties, ${ }^{12,13}$ we became interested in the combination of some structural features of ellipticine-type and amonafide-type 
agents, in continuation of our previous studies aimed at the synthesis and antitumor activity of heterocycle-annulated carbazoles. ${ }^{14,15}$ Here, we wish to report on the preparation of a series of new tetra- and pentacyclic compounds with a carbazole-2,3-dicarboximide core structure and their in-vitro tumor cell-growth inhibitory activity.

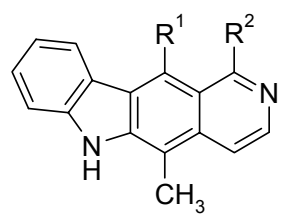

ellipticine $\quad \mathrm{R}^{1}=\mathrm{CH}_{3}, \mathrm{R}^{2}=\mathrm{H}$ olivacine $\quad \mathrm{R}^{1}=\mathrm{H}, \quad \mathrm{R}^{2}=\mathrm{CH}_{3}$

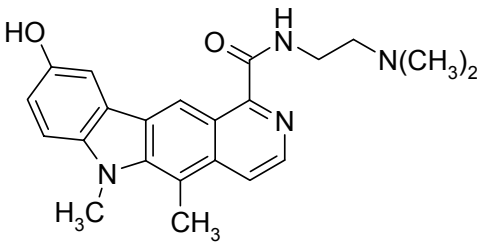

S16020-2

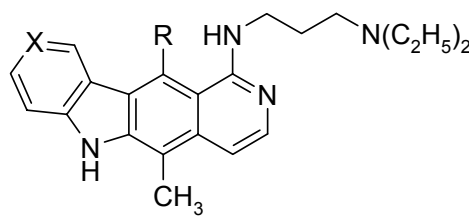

retelliptine $\quad \mathrm{X}=\mathrm{CH}_{3} \mathrm{O}-\mathrm{C}, \mathrm{R}=\mathrm{CH}_{3}$ pazelliptine $\mathrm{X}=\mathrm{N}, \mathrm{R}=\mathrm{H}$

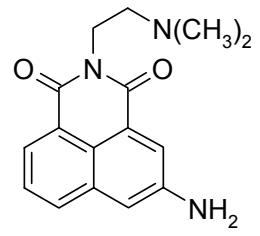

amonafide

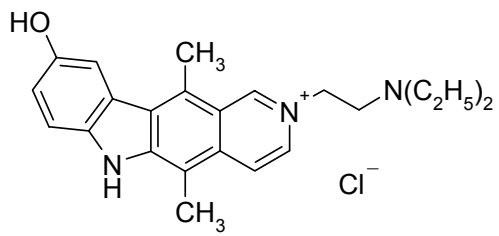

datelliptium

azonafide

\section{Scheme 1}

\section{Results and Discussion}

The easily accessible diester $\mathbf{1 a}^{17}$ (Scheme 2) was chosen as a synthon featuring the 1,4dimethylcarbazole motif of ellipticine as well as the required functional groups which should be suitable for the construction of an imide unit. Initial experiments showed that $\mathbf{1 a}$ can be transformed into a $\mathrm{N}$-substituted carbazole-2,3-dicarboximide derivative simply by heating with an excess of a high-boiling amine such as benzylamine (affording compound 2), but reacts much more sluggishly with low-boiling amines. In the latter case, e.g. for the preparation of the $N$ butyl substituted imide 4, employment of a reactive intermediate is necessary. ${ }^{18}$ For this purpose, the $N$-aminoimide $\mathbf{3}^{19}$ which is easily formed on treatment of $\mathbf{1 a}$ with hydrazine hydrate, can be conveniently used as an "anhydride equivalent", thus giving 4 in good yield upon refluxing in excess $n$-butylamine. The formation of the five-membered cyclic imide structure is clearly evidenced by the characteristic IR absorption bands of the products at $1740-1750 \mathrm{~cm}^{-1}$ and 1680-1700 $\mathrm{cm}^{-1}$, apart from their mass spectra and microanalytical data. For the preparation of the $N$-unsubstituted parent compound $\mathbf{5}$, heating of $\mathbf{1 a}$ in formamide/formic acid was found to be the method of choice, affording 5 in $88 \%$ yield. Introduction of the desired basic side chain was accomplished by prolonged heating of the diester 1a with excess $N, N$-diethyl-1,3- 
propanediamine in DMSO solution under argon atmosphere. This method, which is similar to that described for the preparation of carbazole-1,2-dicarboximides from the corresponding diesters, ${ }^{13}$ gave the diethylaminopropyl-substituted target compound $\mathbf{6 a}$ in high yield. In an analogous fashion, the mono-methyl-substituted carbazole-2,3-diester $\mathbf{1 b}^{20}$ (lacking the methyl group at position 4) was smoothly cyclized into the imide $\mathbf{6 b}$.
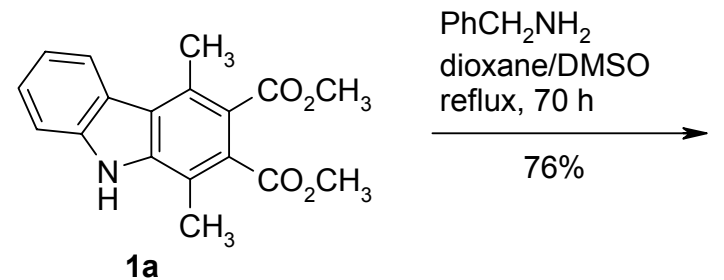

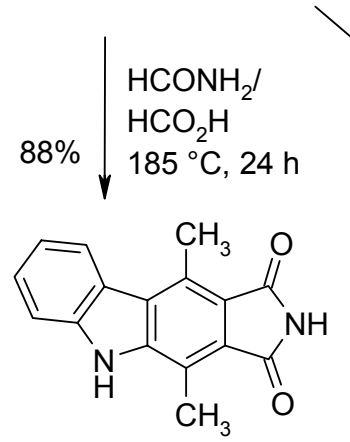

5

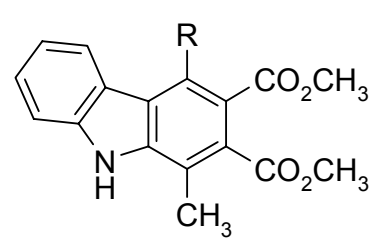

1a $\mathrm{R}=\mathrm{CH}_{3}$

1b $\mathrm{R}=\mathrm{H}$

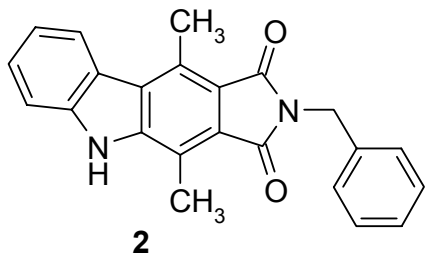

2

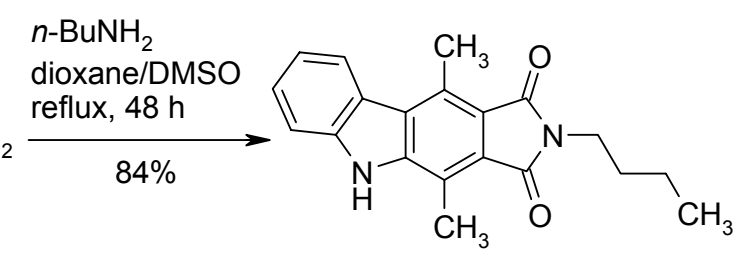

4
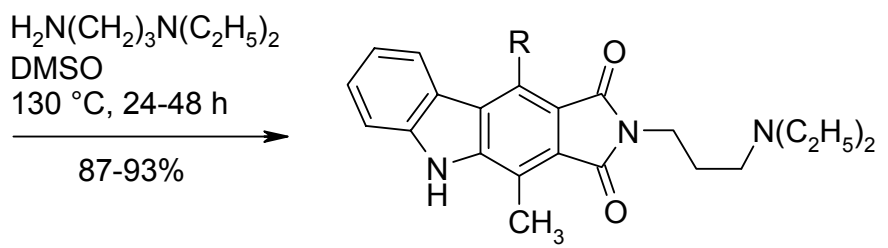

6a $\mathrm{R}=\mathrm{CH}_{3}$ 6b $\mathrm{R}=\mathrm{H}$

\section{Scheme 2}

Enlarging the tetracyclic skeleton into a pentacyclic one, introduction of a three-carbon bridge between the indole nitrogen and the adjacent ring $\mathrm{C}$ was envisaged, thus adding a structural feature of the cytotoxic canthine alkaloids. ${ }^{21}$ The requisite carbazolediesters of type 7 (Scheme 3) had been made available by us previously, ${ }^{15}$ making use of an intramolecular inverse-electron-demand Diels-Alder reaction of appropriately substituted 3-[3-(indol-1yl)propyl]pyridazine-4,5-dicarboxylates. A similar cycloaddition approach had been used by Snyder's group for the construction of bridged $\beta$-carbolines. ${ }^{16}$ Interestingly, the tetracyclic esters 7a,b did not undergo direct cyclization into the target imides by heating with excess primary 
amine under various conditions, despite their steric and electronic similarity to the ester 1a. However, 7a,b could be easily transformed into the pentacyclic $N$-aminoimides 8a,b. Like in the case of compund $\mathbf{3}$, these structures were found to be sufficiently reactive in order to undergo an exchange reaction with excess amine, thus furnishing the desired $N$-substituted imides 9a,b in good yields.<smiles>[R]c1ccc2c(c1)CCN2</smiles><smiles>CCOC(=O)c1c(C)nnc2c1CCCCC2</smiles><smiles>[R]OC(=O)c1c(C(=O)OCC)c2c3c(c1C)c1cc([R])ccc1n3CCC2</smiles>

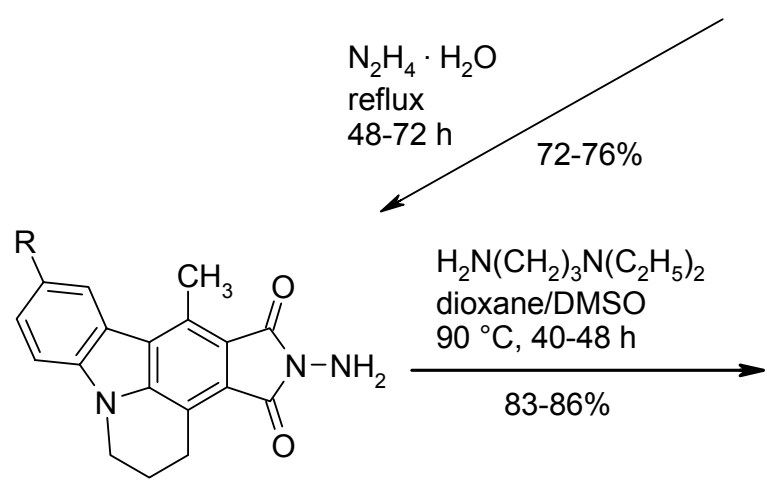

7a $\mathrm{R}=\mathrm{H}$ 7b $\mathrm{R}=\mathrm{CH}_{3} \mathrm{O}$

$8 \mathrm{a} \mathrm{R}=\mathrm{H}$

8b $\mathrm{R}=\mathrm{CH}_{3} \mathrm{O}$

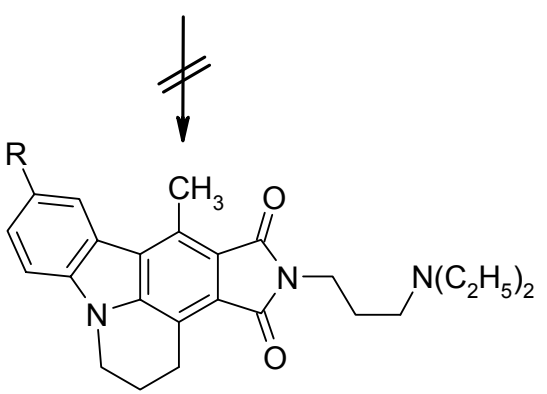

9a $\mathrm{R}=\mathrm{H}$

9b $\mathrm{R}=\mathrm{CH}_{3} \mathrm{O}$

\section{Scheme 3}

The target compounds $\mathbf{6 a}, \mathbf{b}$ and $\mathbf{9 a}, \mathbf{b}$ as well as the $N$-unsubstituted imide $\mathbf{5}$ which represents the core structure were tested in vitro for tumor cell-growth inhibition, using the XTT assay. $^{22}$ The results are summarized in Table 1, they show that $\mathbf{6 a}$ is superior as compared to $\mathbf{6 b}$, the latter lacking the second methyl group at the aromatic scaffold. Likewise, when 9a is compared to its methoxy derivative $\mathbf{9 b}$, the latter structural modification is clearly beneficial. Expectedly, the reference compound $\mathbf{5}$ with an unsubstituted imide nitrogen shows only very weak activity, which demonstrates the importance of the basic side chain in this type of agent. At concentrations lower than $3.16 \mu \mathrm{g} / \mathrm{mL}$, also compounds $\mathbf{6 a}, \mathbf{b}$ and $\mathbf{9 a , b}$ exhibited only weak to moderate effect. 
Table 1. In-vitro antitumor activity (XTT assay; tumor cell growth inhibition in \%) of compounds 5, 6a, 6b, 9a, and $\mathbf{9 b}$ at a fixed sample concentration of $3.16 \mu \mathrm{g} / \mathrm{mL}$

\begin{tabular}{|c|c|c|c|c|}
\hline 5 & $6 \mathbf{a}$ & $6 b$ & 9a & $9 b$ \\
\hline KB-HeLa: $\quad 1 \%$ & KB-HeLa: $99 \%$ & KB-HeLa: $31 \%$ & KB-HeLa: 14\% & KB-HeLa: $35 \%$ \\
\hline SK-OV-3: n.v. & SK-OV-3: $85 \%$ & SK-OV-3: $42 \%$ & SK-OV-3: $12 \%$ & SK-OV-3: $11 \%$ \\
\hline L1210: & L1210: & L1210: & SF-268: $\quad 17 \%$ & SF-268: \\
\hline $\begin{array}{l}\text { MCF-7: } \quad n . v . \\
\text { (n.v.: not valid) }\end{array}$ & MCF-7: & MCF-7: & $\begin{array}{l}\text { NCI-H460: } 47 \% \\
\text { RKOp27: } 21 \%\end{array}$ & $\begin{array}{l}\text { NCI-H460: } 94 \% \\
\text { RKOp27: } 100 \%\end{array}$ \\
\hline
\end{tabular}

Cell lines used:

KB-HeLa: cervical carcinoma

L1210: $\quad$ lymphatic leukemia (mouse)

SF-268: $\quad$ CNS cancer

SK-OV-3: ovarial carcinoma

NCI-H460: non-small-cell lung cancer

MCF-7: mamma carcinoma

RKOp27: colon adenocarcinoma

In conclusion, a series of tetra- and pentacyclic imides with a carbazole skeleton and a basic side chain attached to the imide nitrogen has been made conveniently available either by direct cyclization of a carbazole-2,3-diester with an appropriate primary amine or by a two-step sequence involving an $\mathrm{N}$-aminoimide as a more reactive intermediate. The target compounds show significant tumor cell-growth inhibition in vitro at a concentration of about $10 \mu \mathrm{mol} / \mathrm{L}$.

\section{Experimental Section}

General Procedures. Melting points were determined on a Kofler hot-stage microscope. IR spectra (KBr pellets) were recorded on a Perkin-Elmer 1605 FT-IR instrument. ${ }^{1} \mathrm{H}$ NMR (300 $\mathrm{MHz})$ and ${ }^{13} \mathrm{C}$ NMR $(75 \mathrm{MHz})$ spectra were recorded on a Varian Unityplus 300 spectrometer $(\delta$ values in ppm). Mass spectra were obtained on a Shimadzu QP 5050A DI 50 instrument, highresolution mass spectra (HRMS) were recorded on a Finnigan MAT 8230 spectrometer at the Institute of Organic Chemistry, University of Vienna. For column chromatography, Merck Kieselgel $60 \quad(0.063-0.200 \mathrm{~mm})$ was used. Microanalyses ${ }^{23}$ were performed at the Microanalytical Laboratory, Faculty of Chemistry, University of Vienna.

2-Benzyl-4,10-dimethylpyrrolo[3,4-b]carbazole-1,3(2H,5H)-dione (2). A mixture of dimethyl 1,4-dimethyl-9H-carbazole-2,3-dicarboxylate $(\mathbf{1 a})^{17}(155 \mathrm{mg}, 0.50 \mathrm{mmol})$, benzylamine $(10.0$ $\mathrm{mL}, 92.0 \mathrm{mmol})$, dioxane $(10 \mathrm{~mL})$ and DMSO $(3 \mathrm{~mL})$ was heated under reflux for $70 \mathrm{~h}$. The volatile components were removed under reduced pressure and the residue was recrystallized from $\mathrm{MeOH} / \mathrm{AcOEt}$ to give $2(135 \mathrm{mg}, 76 \%)$ as colorless crystals, mp $313^{\circ} \mathrm{C}$. Anal. Calcd. $\mathrm{C}_{23} \mathrm{H}_{18} \mathrm{~N}_{2} \mathrm{O}_{2}$ : C, 77.95; H, 5.12; N, 7.90. Found: C, 78.33; H, 5.02; N, 7.85. IR (KBr, cm $\left.{ }^{-1}\right) 3378$, 3063, 3032, 2941, 1739, 1687, 1433, 1394, 1337, 749, 730, 698; ${ }^{1} \mathrm{H}$ NMR (DMSO-d $\left.{ }_{6}\right) \delta 12.08$ 
(br s, $1 \mathrm{H}, \mathrm{NH}$, shows positive NOE on irradiation at $2.84 \mathrm{ppm}), 8.24\left(\mathrm{~d}, J_{8-9}=8.1 \mathrm{~Hz}, 1 \mathrm{H}, 9-\mathrm{H}\right.$, shows positive NOE on irradiation at $3.12 \mathrm{ppm}), 7.63\left(\mathrm{~d}, J_{6-7}=8.1 \mathrm{~Hz}, 1 \mathrm{H}, 6-\mathrm{H}\right), 7.53-7.47$ (m, $1 \mathrm{H}, 7-\mathrm{H}), 7.37-7.24$ (m, 6H, 8-H, phenyl-H), $4.70\left(\mathrm{~s}, 2 \mathrm{H}, \mathrm{NCH}_{2}\right), 3.12\left(\mathrm{~s}, 3 \mathrm{H}, 10-\mathrm{CH}_{3}\right), 2.84(\mathrm{~s}$, 3H, 4- $\left.\mathrm{CH}_{3}\right)$; MS m/z: $354\left(\mathrm{M}^{+}, 100 \%\right), 263$ (47), 248 (24), 221 (13), 192 (30), 191 (27), 165 (15), 106 (23), 104 (24), 91 (89), 78 (39), 77 (62), 65 (53), 51 (59).

2-Butyl-4,10-dimethylpyrrolo[3,4-b]carbazole-1,3(2H,5H)-dione (4). A mixture of 2-amino4,10-dimethylpyrrolo[3,4-b]carbazole-1,3(2H,5H)-dione $\quad(3)^{19} \quad(100 \mathrm{mg}, \quad 0.36 \mathrm{mmol}), \quad n$ butylamine $(10.0 \mathrm{~mL}, 101.0 \mathrm{mmol})$, dioxane $(10 \mathrm{~mL})$ and DMSO $(3 \mathrm{~mL})$ was heated under reflux for $48 \mathrm{~h}$. The volatile components were removed under reduced pressure and the residue was purified by short-column chromatography (light petroleum/AcOEt, 4+1), followed by recrystallization from 2-PrOH to afford 4 (98 $\mathrm{mg}, 84 \%)$ as pale yellow crystals, $\mathrm{mp} 286-288^{\circ} \mathrm{C}$. Anal. Calcd. $\mathrm{C}_{20} \mathrm{H}_{20} \mathrm{~N}_{2} \mathrm{O}_{2} \cdot 0.25 \mathrm{H}_{2} \mathrm{O}: \mathrm{C}, 73.94 ; \mathrm{H}, 6.36 ; \mathrm{N}, 8.62$. Found: C, 74.14; H, 6.19; N, 8.56. IR (KBr, cm $\left.{ }^{-1}\right) 3347,2957,1748,1677,1435,1401,1367,747,729 ;{ }^{1} \mathrm{H}$ NMR (DMSO- $d_{6}$ ) $\delta 11.93$ (br s, $1 \mathrm{H}, \mathrm{NH}), 8.23\left(\mathrm{~d}, J_{8-9}=8.1 \mathrm{~Hz}, 1 \mathrm{H}, 9-\mathrm{H}\right.$, shows positive NOE on irradiation at $3.11 \mathrm{ppm}), 7.63\left(\mathrm{~d}, J_{6-7}=8.4 \mathrm{~Hz}, 1 \mathrm{H}, 6-\mathrm{H}\right), 7.54-7.47(\mathrm{~m}, 1 \mathrm{H}, 7-\mathrm{H}), 7.32-7.24(\mathrm{~m}, 1 \mathrm{H}, 8-\mathrm{H})$, 3.52 (t, $\left.J=7.3 \mathrm{~Hz}, 2 \mathrm{H}, \mathrm{NCH}_{2} \mathrm{CH}_{2} \mathrm{CH}_{2} \mathrm{CH}_{3}\right), 3.11\left(\mathrm{~s}, 3 \mathrm{H}, 10-\mathrm{CH}_{3}\right), 2.84\left(\mathrm{~s}, 3 \mathrm{H}, 4-\mathrm{CH}_{3}\right), 1.58$ (quint, $\left.J=7.3 \mathrm{~Hz}, 2 \mathrm{H}, \mathrm{NCH}_{2} \underline{\mathrm{C}}_{2} \mathrm{CH}_{2} \mathrm{CH}_{3}\right), 1.37-1.24\left(\mathrm{~m}, 2 \mathrm{H}, \mathrm{NCH}_{2} \mathrm{CH}_{2} \mathrm{C}_{2} \mathrm{CH}_{3}\right.$ ), 0.91 (t, $\left.J=7.3 \mathrm{~Hz}, 3 \mathrm{H}, \mathrm{NCH}_{2} \mathrm{CH}_{2} \mathrm{CH}_{2} \mathrm{CH}_{3}\right)$; MS m/z: $320\left(\mathrm{M}^{+}, 66 \%\right), 278$ (51), 277 (100), 264 (26), 193 (16), 192 (15), 191 (10); HRMS Calcd. $\mathrm{C}_{20} \mathrm{H}_{20} \mathrm{~N}_{2} \mathrm{O}_{2}: 320.1525$. Found: 320.1519.

4,10-Dimethylpyrrolo[3,4-b]carbazole-1,3(2H,5H)-dione (5). A mixture of dimethyl 1,4dimethyl-9H-carbazole-2,3-dicarboxylate (1a) ${ }^{17}(311 \mathrm{mg}, 1.00 \mathrm{mmol})$, formic acid $(2 \mathrm{~mL})$ and formamide $(30 \mathrm{~mL})$ was heated to $185^{\circ} \mathrm{C}$ for $24 \mathrm{~h}$. After cooling, the precipitate was collected by filtration, washed with water and dried to give $5(235 \mathrm{mg}, 88 \%)$ as pale yellow crystals, $\mathrm{mp}>$ $350^{\circ} \mathrm{C}$. Anal. Calcd. $\mathrm{C}_{16} \mathrm{H}_{12} \mathrm{~N}_{2} \mathrm{O}_{2} \cdot 0.2 \mathrm{H}_{2} \mathrm{O}$ : C, 71.74; H, 4.67; N, 10.46. Found: C, 71.66; H, 4.74; N, 10.31. IR (KBr, cm $\left.{ }^{-1}\right) 3327,3170,3049,1744,1700,1365,1330,761,730,652 ;{ }^{1} \mathrm{H}$ NMR (DMSO-d $\left.)_{6}\right) \delta 12.01$ (br s, 1H, NH), 10.88 (br s, 1H, NH), 8.23 (d, $J_{8-9}=8.1 \mathrm{~Hz}, 1 \mathrm{H}, 9-\mathrm{H}$, shows positive NOE on irradiation at $3.10 \mathrm{ppm}), 7.62\left(\mathrm{~d}, J_{6-7}=8.1 \mathrm{~Hz}, 1 \mathrm{H}, 6-\mathrm{H}\right), 7.53-7.47(\mathrm{~m}$, $1 \mathrm{H}, 7-\mathrm{H}), 7.30-7.24(\mathrm{~m}, 1 \mathrm{H}, 8-\mathrm{H}), 3.10\left(\mathrm{~s}, 3 \mathrm{H}, 10-\mathrm{CH}_{3}\right), 2.82\left(\mathrm{~s}, 3 \mathrm{H}, 4-\mathrm{CH}_{3}\right) ;{ }^{13} \mathrm{C}$ NMR (DMSO$\left.d_{6}\right) \delta 170.4,170.0,141.9,140.8,131.4,126.4,125.8,124.1,123.0,122.8,120.1,119.4,118.8$, 111.8, 14.2, 11.6; MS m/z: $264\left(\mathrm{M}^{+}, 100 \%\right), 193$ (38), 95 (50), 83 (54), 69 (32), 57 (38), 55 (32).

2-[3-(Diethylamino)propyl]-4,10-dimethylpyrrolo[3,4-b]carbazole-1,3(2H,5H)-dione (6a). A solution of dimethyl 1,4-dimethyl-9H-carbazole-2,3-dicarboxylate (1a) ${ }^{17}$ (311 mg, $\left.1.00 \mathrm{mmol}\right)$ and $N, N$-diethyl-1,3-propanediamine $(1302 \mathrm{mg}, 10.00 \mathrm{mmol})$ in DMSO $(5 \mathrm{~mL})$ was stirred at $130^{\circ} \mathrm{C}$ for $24 \mathrm{~h}$ under an argon atmosphere. Another portion of $N, N$-diethyl-1,3-propanediamine (1302 $\mathrm{mg}, 10.00 \mathrm{mmol}$ ) was added and heating was continued for further $24 \mathrm{~h}$. The volatile components were removed under reduced pressure and the residue was triturated with $\mathrm{MeOH}$. The almost colorless, crystalline material was collected by filtration and the filtrate was subjected to column chromatography (AcOEt/light petroleum/ $\left.\mathrm{Et}_{3} \mathrm{~N}, 95+95+10\right)$ to afford another portion of the product. The combined crops were recrystallized from AcOEt to give 6a $(337 \mathrm{mg}$, $87 \%$ ) as colorless crystals, mp $232-235^{\circ} \mathrm{C}$. Anal. Calcd. $\mathrm{C}_{23} \mathrm{H}_{27} \mathrm{~N}_{3} \mathrm{O}_{2} \cdot 0.6 \mathrm{H}_{2} \mathrm{O}: \mathrm{C}, 71.15 ; \mathrm{H}$, 
7.32; N, 10.82. Found: C, 71.19; H, 7.02; N, 10.77. IR (KBr, $\left.\mathrm{cm}^{-1}\right) 3343,2968,1745,1676$, 1437, 1401, 1364, 1032, 746, 730, 628; ${ }^{1} \mathrm{H}$ NMR (DMSO-d $) \delta 12.03$ (br s, $\left.1 \mathrm{H}, \mathrm{NH}\right), 8.23\left(\mathrm{~d}, J_{8-}\right.$ $9=8.1 \mathrm{~Hz}, 1 \mathrm{H}, 9-\mathrm{H}), 7.62\left(\mathrm{~d}, J_{6-7}=8.1 \mathrm{~Hz}, 1 \mathrm{H}, 6-\mathrm{H}\right), 7.54-7.46(\mathrm{~m}, 1 \mathrm{H}, 7-\mathrm{H}), 7.31-7.24(\mathrm{~m}$, $1 \mathrm{H}, 8-\mathrm{H}), 3.54$ (t, $\left.J=7.2 \mathrm{~Hz}, 2 \mathrm{H}, \mathrm{NCH}_{2} \mathrm{CH}_{2} \mathrm{CH}_{2} \mathrm{~N}\left(\mathrm{CH}_{2} \mathrm{CH}_{3}\right)_{2}\right), 3.11$ (s, 3H, 10- $\left.\mathrm{CH}_{3}\right), 2.83$ (s, $\left.3 \mathrm{H}, \quad 4-\mathrm{CH}_{3}\right), \quad 2.46-2.37 \quad\left(\mathrm{~m}, \quad 6 \mathrm{H}, \quad \mathrm{NCH}_{2} \mathrm{CH}_{2} \mathrm{CH}_{2} \mathrm{~N}\left(\mathrm{C}_{2} \mathrm{CH}_{3}\right)_{2}\right), \quad 1.74-1.63 \quad(\mathrm{~m}, \quad 2 \mathrm{H}$, $\left.\mathrm{NCH}_{2} \underline{\mathrm{C}}_{2} \mathrm{CH}_{2} \mathrm{~N}\left(\mathrm{CH}_{2} \mathrm{CH}_{3}\right)_{2}\right), 0.90\left(\mathrm{t}, J=7.2 \mathrm{~Hz}, 6 \mathrm{H}, \mathrm{N}\left(\mathrm{CH}_{2} \mathrm{CH}_{3}\right)_{2}\right) ;{ }^{13} \mathrm{C} \mathrm{NMR}$ (DMSO- $\left.d_{6}\right) \delta$ $168.8,168.4,141.6,140.7,131.3,126.2,124.6,123.8,122.9,122.6,120.0,118.8,118.2,111.7$, 50.0, 46.1 35.4, 25.7, 14.2, 11.7, 11.6; MS m/z: 377 (M+2\%), 348 (5), 305 (3), 277 (7), 193 (3), 192 (3), 181 (4), 139 (3), 112 (3), 87 (5), 86 (100), 84 (8), 72 (26), 58 (21); HRMS Calcd. $\mathrm{C}_{23} \mathrm{H}_{27} \mathrm{~N}_{3} \mathrm{O}_{2}: 377.2103$. Found: 377.2112 .

2-[3-(Diethylamino)propyl]-4-methylpyrrolo[3,4-b]carbazole-1,3(2H,5H)-dione (6b). A solution of dimethyl 1-methyl-9H-carbazole-2,3-dicarboxylate (1b) ${ }^{20}(297 \mathrm{mg}, 1.00 \mathrm{mmol})$ and $N, N$-diethyl-1,3-propanediamine $(1302 \mathrm{mg}, 10.00 \mathrm{mmol})$ in DMSO $(5 \mathrm{~mL})$ was stirred at $130^{\circ} \mathrm{C}$ for $24 \mathrm{~h}$ under an argon atmosphere. The volatile components were removed under reduced pressure and the residue was purified by column chromatography (AcOEt/light petroleum/Et ${ }_{3} \mathrm{~N}$, 95+95+10), followed by recrystallization from AcOEt to give $6 \mathbf{b}(338 \mathrm{mg}, 93 \%)$ as almost colorless crystals, mp $203-207^{\circ} \mathrm{C}$. Anal. Calcd. $\mathrm{C}_{22} \mathrm{H}_{25} \mathrm{~N}_{3} \mathrm{O}_{2}$ : C, 72.70; H, 6.93; N, 11.56. Found: C, 72.91; H, 7.15; N, 11.64. IR (KBr, cm ${ }^{-1}$ ) 3332, 2968, 2800, 1752, 1689, 1460, 1397, 1361, 1248, 1040, 750, 730; ${ }^{1} \mathrm{H}$ NMR (DMSO- $d_{6}$ ) $\delta 12.01$ (br s, $\left.1 \mathrm{H}, \mathrm{NH}\right), 8.46$ (s, 1H, 10-H), 8.29 (d, $\left.J_{8-9}=7.8 \mathrm{~Hz}, 1 \mathrm{H}, 9-\mathrm{H}\right), 7.59\left(\mathrm{~d}, J_{6-7}=8.4 \mathrm{~Hz}, 1 \mathrm{H}, 6-\mathrm{H}\right), 7.52-7.46(\mathrm{~m}, 1 \mathrm{H}, 7-\mathrm{H}), 7.28-7.22(\mathrm{~m}$, $1 \mathrm{H}, 8-\mathrm{H}), 3.56\left(\mathrm{t}, J=7.4 \mathrm{~Hz}, 2 \mathrm{H}, \mathrm{NCH}_{2} \mathrm{CH}_{2} \mathrm{CH}_{2} \mathrm{~N}\left(\mathrm{CH}_{2} \mathrm{CH}_{3}\right)_{2}\right), 2.86$ (s, 3H, 4-CH $)_{3}, 2.45-2.35$ (m, 6H, $\left.\mathrm{NCH}_{2} \mathrm{CH}_{2} \mathrm{CH}_{2} \mathrm{~N}\left(\mathrm{CH}_{2} \mathrm{CH}_{3}\right)_{2}\right), 1.75-1.64$ (m, 2H, $\left.\mathrm{NCH}_{2} \underline{\mathrm{C}}_{2} \mathrm{CH}_{2} \mathrm{~N}\left(\mathrm{CH}_{2} \mathrm{CH}_{3}\right)_{2}\right), 0.89$ (t, $J$ $\left.=7.1 \mathrm{~Hz}, 6 \mathrm{H}, \mathrm{NCH}_{2} \mathrm{CH}_{2} \mathrm{CH}_{2} \mathrm{~N}\left(\mathrm{CH}_{2} \mathrm{CH}_{3}\right)_{2}\right) ;{ }^{13} \mathrm{C}$ NMR (DMSO-d 6 ) $\delta 169.0,168.1,142.2,140.9$, 127.2, 124.8, 124.7, 122.6, 122.2, 121.3, 121.1, 120.1, 114.0, 111.8, 50.0, 46.1, 35.7, 25.7, 12.0, 11.6; MS m/z: 363 (M+, 2\%), 348 (2), 334 (7), 291 (5), 263 (7), 179 (3), 178 (4), 174 (7), 132 (2), 112 (4), 87 (7), 86 (100), 84 (7), 72 (32), 58 (12).

11-Amino-9-methyl-2,3-dihydropyrido[1,2,3-lm]pyrrolo[3,4-b]carbazole-10,12(1H, $11 H)$ dione (8a). A mixture of diethyl 1-methyl-5,6-dihydro-4H-pyrido[3,2,1-jk]carbazole-2,3dicarboxylate (7a) ${ }^{15}(117 \mathrm{mg}, 0.32 \mathrm{mmol})$ and hydrazine monohydrate $(4.0 \mathrm{~mL}, 82.0 \mathrm{mmol})$ was refluxed for $48 \mathrm{~h}$. After cooling, EtOH ( $4 \mathrm{~mL})$ was added and the precipitate was collected by filtration and washed several times with boiling $\mathrm{MeOH}$ to give $\mathbf{8 a}$ (76 $\mathrm{mg}, 76 \%$ ) as pale yellow crystals, mp $295-300^{\circ} \mathrm{C}$ (decomp). Anal. Calcd. $\mathrm{C}_{18} \mathrm{H}_{15} \mathrm{~N}_{3} \mathrm{O}_{2} \cdot 0.4 \mathrm{H}_{2} \mathrm{O}: \mathrm{C}, 69.17$; H, 5.10; N, 13.44. Found: C, 69.14; H, 4.95; N, 13.44. IR (KBr, cm $\left.{ }^{-1}\right) 3322,3046,2929,1756,1703,1617$, 1518, 1413, 1333, 1303, 1253, 748; ${ }^{1} \mathrm{H}$ NMR (DMSO-d $\left.d_{6}\right) \delta 8.29\left(\mathrm{~d}, J_{7-8}=7.8 \mathrm{~Hz}, 1 \mathrm{H}, 8-\mathrm{H}\right), 7.72$ $\left(\mathrm{d}, J_{5-6}=8.1 \mathrm{~Hz}, 1 \mathrm{H}, 5-\mathrm{H}\right), 7.64-7.54(\mathrm{~m}, 1 \mathrm{H}, 6-\mathrm{H}), 7.40-7.30(\mathrm{~m}, 1 \mathrm{H}, 7-\mathrm{H}), 4.85\left(\mathrm{~s}, 2 \mathrm{H}, \mathrm{NH}_{2}\right)$, $4.36\left(\mathrm{t}, J_{2-3}=5.7 \mathrm{~Hz}, 2 \mathrm{H}, 3-\mathrm{H}\right), 3.38\left(\mathrm{t}, J_{1-2}=6.1 \mathrm{~Hz}, 2 \mathrm{H}, 1-\mathrm{H}\right), 3.14\left(\mathrm{~s}, 3 \mathrm{H}, \mathrm{CH}_{3}\right), 2.36-2.20(\mathrm{~m}$, 2H, 2-H); MS m/z: 305 (M+, 100\%), 289 (23), 260 (50), 244 (22), 232 (33), 217 (50), 204 (30), 190 (22), 163 (16), 152 (83), 145 (40), 138 (18), 123 (28), 115 (17), 109 (76), 102 (35), 95 (39), 82 (16); HRMS Calcd. $\mathrm{C}_{18} \mathrm{H}_{15} \mathrm{~N}_{3} \mathrm{O}_{2}$ : 305.1164. Found: 305.1171. 


\section{1-Amino-7-methoxy-9-methyl-2,3-dihydropyrido[1,2,3-lm]pyrrolo[3,4-b]carbazole-}

10,12(1H,11H)-dione (8b). A mixture of diethyl 10-methoxy-1-methyl-5,6-dihydro-4Hpyrido[3,2,1-jk]carbazole-2,3-dicarboxylate $(\mathbf{7 b})^{15}(121 \mathrm{mg}, 0.31 \mathrm{mmol})$ and hydrazine monohydrate $(4.0 \mathrm{~mL}, 5.0 \mathrm{mmol})$ was refluxed for $72 \mathrm{~h}$. After cooling, EtOH $(4 \mathrm{~mL})$ was added and the precipitate was collected by filtration and washed with EtOH to give $\mathbf{8 b}(77 \mathrm{mg}, 72 \%)$ as pale yellow crystals, mp 247-252 ${ }^{\circ} \mathrm{C}$. Anal. Calcd. $\mathrm{C}_{19} \mathrm{H}_{17} \mathrm{~N}_{3} \mathrm{O}_{3} \cdot 0.5 \mathrm{H}_{2} \mathrm{O}: \mathrm{C}, 66.27 ; \mathrm{H}, 5.27 ; \mathrm{N}$, 12.20. Found: C, 66.14; H, 5.13; N, 12.01. IR (KBr, cm $\left.{ }^{-1}\right) 3334,2942,2832,1748,1696,1612$, $1481,1411,1283,1226,1144,1033,748,677 ;{ }^{1} \mathrm{H}$ NMR (DMSO- $\left.d_{6}\right) \delta 7.68\left(\mathrm{~d}, J_{6-8}=2.4 \mathrm{~Hz}\right.$, $1 \mathrm{H}, 8-\mathrm{H}), 7.62\left(\mathrm{~d}, J_{5-6}=9.0 \mathrm{~Hz}, 1 \mathrm{H}, 5-\mathrm{H}\right), 7.22\left(\mathrm{dd}, J_{5-6}=9.0 \mathrm{~Hz}, J_{6-8}=2.4 \mathrm{~Hz}, 1 \mathrm{H}, 6-\mathrm{H}\right), 4.81$ (br s, $\left.2 \mathrm{H}, \mathrm{NH}_{2}\right), 4.28\left(\mathrm{t}, J_{2-3}=5.8 \mathrm{~Hz}, 2 \mathrm{H}, 3-\mathrm{H}\right), 3.88\left(\mathrm{~s}, 3 \mathrm{H}, \mathrm{OCH}_{3}\right), 3.32\left(\mathrm{t}, J_{1-2}=6.0 \mathrm{~Hz}, 2 \mathrm{H}\right.$, 1-H), 3.09 (s, 3H, 9-CH $\mathrm{CH}_{3}$, 2.30-2.16 (m, 2H, 2-H); MS m/z: 335 (M+1, 100\%), 320 (49), 290 (26), 275 (9), 262 (9), 247 (11), 234 (12), 204 (10), 168 (12), 138 (23), 124 (31), 102 (36), 95 (26), 82 (11); HRMS Calcd. $\mathrm{C}_{19} \mathrm{H}_{17} \mathrm{~N}_{3} \mathrm{O}_{3}$ : 335.1270. Found: 335.1261 .

11-[3-(Diethylamino)propyl]-9-methyl-2,3-dihydropyrido[1,2,3-lm]pyrrolo[3,4-b]carbazole10,12(1H,11H)-dione (9a). A mixture of $8 \mathbf{a}$ (98 $\mathrm{mg}, 0.32 \mathrm{mmol}), \quad N, N$-diethyl-1,3propanediamine $(2.0 \mathrm{~mL}, 12.7 \mathrm{mmol})$, dioxane $(2 \mathrm{~mL})$ and DMSO $(2 \mathrm{~mL})$ was heated in a closed vessel to $90^{\circ} \mathrm{C}$ for $48 \mathrm{~h}$ under an argon atmosphere. The volatile components were removed under reduced pressure and the residue was triturated with $\mathrm{MeOH}$. The crystalline material was collected by filtration and washed with $\mathrm{MeOH}$ to give 9a (108 $\mathrm{mg}, 83 \%)$ as colorless crystals, mp 174- $177^{\circ}$ C. Anal. Calcd. $\mathrm{C}_{25} \mathrm{H}_{29} \mathrm{~N}_{3} \mathrm{O}_{2}$ : C, 74.41; H, 7.24; N, 10.41. Found: C, 74.19; H, 7.07; $\mathrm{N}, 10.47$. IR (KBr, cm $\left.{ }^{-1}\right) 2967,2798,1746,1684,1416,1375,1332,747 ;{ }^{1} \mathrm{H}$ NMR (DMSO- $d_{6}$ ) $\delta 8.28\left(\mathrm{~d}, J_{7-8}=7.8 \mathrm{~Hz}, 1 \mathrm{H}, 8-\mathrm{H}\right), 7.71\left(\mathrm{~d}, J_{5-6}=8.1 \mathrm{~Hz}, 1 \mathrm{H}, 5-\mathrm{H}\right.$, shows positive NOE on irradiation at $4.34 \mathrm{ppm}), 7.62-7.53(\mathrm{~m}, 1 \mathrm{H}, 6-\mathrm{H}), 7.38-7.29(\mathrm{~m}, 1 \mathrm{H}, 7-\mathrm{H}), 4.34\left(\mathrm{t}, J_{2-3}=5.8 \mathrm{~Hz}\right.$, $2 \mathrm{H}, 3-\mathrm{H}), 3.57$ (t, $\left.J=7.2 \mathrm{~Hz}, 2 \mathrm{H}, \mathrm{NCH}_{2} \mathrm{CH}_{2} \mathrm{CH}_{2} \mathrm{~N}\left(\mathrm{CH}_{2} \mathrm{CH}_{3}\right)_{2}\right), 3.37$ (t, $\left.J_{1-2}=6.0 \mathrm{~Hz}, 2 \mathrm{H}, 1-\mathrm{H}\right)$,

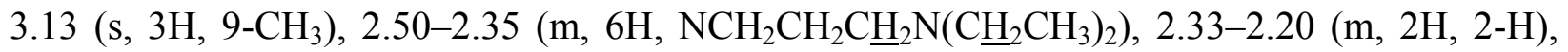
1.79-1.62 (m, 2H, $\left.\quad \mathrm{NCH}_{2} \mathrm{CH}_{2} \mathrm{CH}_{2} \mathrm{~N}_{(}\left(\mathrm{CH}_{2} \mathrm{CH}_{3}\right)_{2}\right), \quad 0.91 \quad(\mathrm{t}, \quad J=7.0 \mathrm{~Hz}, \quad 6 \mathrm{H}$, $\left.\mathrm{NCH}_{2} \mathrm{CH}_{2} \mathrm{CH}_{2} \mathrm{~N}\left(\mathrm{CH}_{2} \underline{\mathrm{C}}_{3}\right)_{2}\right)$; MS m/z: $403\left(\mathrm{M}^{+}, 2 \%\right), 374$ (9), 331 (4), 303 (5), 218 (6), 112 (6), 86 (100), 72 (21), 58 (12).

\section{1-[3-(Diethylamino)propyl]-7-methoxy-9-methyl-2,3-dihydropyrido[1,2,3-lm]pyrrolo[3,4-} b]carbazole-10,12(1H,11H)-dione (9b). A mixture of $8 \mathbf{b}(100 \mathrm{mg}, 0.30 \mathrm{mmol}), N, N$-diethyl-1,3propanediamine $(2.0 \mathrm{~mL}, 12.7 \mathrm{mmol})$, dioxane $(2 \mathrm{~mL})$ and DMSO $(2 \mathrm{~mL})$ was heated in a closed vessel to $90^{\circ} \mathrm{C}$ for $40 \mathrm{~h}$ under an argon atmosphere. The volatile components were removed under reduced pressure and the residue was triturated with $\mathrm{MeOH}$. The crystalline material was collected by filtration and washed with $\mathrm{MeOH}$ to give $\mathbf{9 b}(113 \mathrm{mg}, 86 \%)$ as pale yellow crystals, mp 173-177 ${ }^{\circ}$ C. Anal. Calcd. $\mathrm{C}_{26} \mathrm{H}_{31} \mathrm{~N}_{3} \mathrm{O}_{3} \cdot 0.4 \mathrm{H}_{2} \mathrm{O}$ : C, 70.85; H, 7.27; N, 9.53. Found: C, 70.81; H, 7.07; N, 9.78. IR (KBr, cm $\left.{ }^{-1}\right)$ 2966, 2938, 2804, 1745, 1687, 1484, 1411, 1310, 1230, 1145, 1030, 832; ${ }^{1} \mathrm{H}$ NMR (DMSO- $\left.d_{6}\right) \delta 7.64\left(\mathrm{~d}, J_{6-8}=2.2 \mathrm{~Hz}, 1 \mathrm{H}, 8-\mathrm{H}\right), 7.60\left(\mathrm{~d}, J_{5-6}=8.8 \mathrm{~Hz}\right.$, $1 \mathrm{H}, 5-\mathrm{H}), 7.21\left(\mathrm{dd}, J_{5-6}=8.8 \mathrm{~Hz}, J_{6-8}=2.2 \mathrm{~Hz}, 1 \mathrm{H}, 6-\mathrm{H}\right), 4.25\left(\mathrm{t}, J_{2-3}=5.5 \mathrm{~Hz}, 2 \mathrm{H}, 3-\mathrm{H}\right), 3.87$

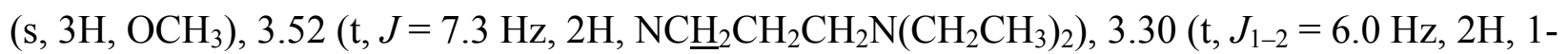
$\mathrm{H}), 3.05$ (s, 3H, 9- $\left.\mathrm{CH}_{3}\right), 2.50-2.33\left(\mathrm{~m}, 6 \mathrm{H}, \mathrm{NCH}_{2} \mathrm{CH}_{2} \mathrm{C}_{2} \mathrm{~N}_{\left(\mathrm{CH}_{2} \mathrm{CH}_{3}\right)}\right.$ ), 2.30-2.12 (m, 2H, 2- 
$\mathrm{H}), \quad 1.77-1.59\left(\mathrm{~m}, \quad 2 \mathrm{H}, \quad \mathrm{NCH}_{2} \mathrm{CH}_{2} \mathrm{CH}_{2} \mathrm{~N}\left(\mathrm{CH}_{2} \mathrm{CH}_{3}\right)_{2}\right), \quad 0.91 \quad(\mathrm{t}, \quad J=7.0 \mathrm{~Hz}, \quad 6 \mathrm{H}$, $\left.\mathrm{NCH}_{2} \mathrm{CH}_{2} \mathrm{CH}_{2} \mathrm{~N}\left(\mathrm{CH}_{2} \underline{\mathrm{C}}_{3}\right)_{2}\right)$; MS m/z: $433\left(\mathrm{M}^{+}, 2 \%\right), 404$ (8), 361 (3), 333 (6), 112 (7), 86 (100), 72 (20), 58 (11); HRMS Calcd. $\mathrm{C}_{26} \mathrm{H}_{31} \mathrm{~N}_{3} \mathrm{O}_{3}$ : 433.2365. Found: 433.2374.

\section{Acknowledgements}

We are grateful to Zentaris $\mathrm{GmbH}$ (Frankfurt/Main, Germany) for in-vitro testing of the compounds for antitumor activity.

\section{References}

1. Gribble, G. W. In The Alkaloids, Brossi, A., Ed., Academic Press: New York, 1990, Vol. 39, p 239.

2. Ohashi, M.; Oki, T. Exp. Opin. Ther. Patents 1996, 6, 1285.

3. Ducrocq, C.; Wendling, F.; Tourbez-Perrin, M.; Rivalle, C.; Tambourin, P.; Pochon, F.; Bisagni, E.; Chermann, J. C. J. Med. Chem. 1980, 23, 1212.

4. Vilarem, M. J.; Charcosset, J. Y.; Primaux, F.; Gras, M. P.; Calvo, F.; Larsen, C. J. Cancer Res. 1985, 45, 3906.

5. (a) Auclair, C.; Pierre, A.; Voisin, E.; Pepin, O.; Cros, S.; Colas, C.; Saucier, J. M.; Verschuere, B.; Gros, P.; Paoletti, C. Cancer Res. 1987, 47, 6254. (b) Auclair, C.; Polard, V.; Maksimenko, A. PCT Int. Appl. 135538, 2007; Chem. Abstr. 2007, 148, 24396.

6. (a) Guillonneau, C.; Pierré, A.; Charton, Y.; Guilbaud, N.; Kraus-Berthier, L.; Léonce, S.; Michel, A.; Bisagni, E.; Atassi, G. J. Med. Chem. 1999, 42, 2191. (b) Malonne, H.; Farinelle, S.; Decaestecker, C.; Gordower, L.; Fontaine, J.; Chaminade, F.; Saucier, J.-M.; Atassi, G.; Kiss, R. Clin. Cancer Res. 2000, 6, 3774.

7. Pommier, Y. Cancer Chemother. Pharmacol. 1993, 32, 103.

8. (a) Braña, M. F.; Sanz, A. M.; Castellano, J. M.; Roldán C. M.; Roldán, C. Eur. J. Med. Chem. 1981, 16, 207. (b) Constanza, M. E.; Berry, D.; Henderson, I. C.; Ratain, M. J.; Wu, K.; Shapiro, C.; Duggan, D.; Kalra, J.; Berkowitz I.; Lyss, A. P. Clin. Cancer Res. 1995, 699.

9. (a) Sami, S. M.; Dorr, R. T.; Alberts D. S.; Remers, W. A. J. Med. Chem. 1993, 36, 765. (b) Remers, W. A.; Dorr, R. T.; Sami, S. M.; Alberts, D. S.; Bear, S.; Mayr, C. A.; Solyom, A. M. Curr. Topics Med. Chem. 1997, 2, 45.

10. Chau, M.; Christensen, J. L.; Ajami, A. M.; Capizzi, R. L. Leukemia Res. 2008, 32, 465.

11. Pierré, A.; Léonce, S.; Pérez, V.; Atassi, G. Cancer Chemother. Pharmacol. 1998, 42, 454.

12. Nagai, T.; Myokan, I.; Funaki, K.; Ohta, K.; Taya, N.; Miyabara, S.; Shibata, M.; Mikami, H.; Hori, T. Ger. Offen. 4034 687, 1991; Chem. Abstr. 1991, 115, 279992. 
13. Joseph, B.; Facompré, M.; Da Costa, H.; Routier, S.; Mérour, J.-Y.; Colson, P.; Houssier, C.; Bailly, C. Bioorg. Med. Chem. 2001, 9, 1533.

14. (a) Haider, N.; Jbara, R.; Khadami, F.; Wanko, R. Heterocycles 1998, 48, 1609. (b) Haider, N.; Käferböck, J.; Mátyus, P. Heterocycles 1999, 51, 2703. (c) Fidesser, E.; Haider, N.; Jbara, R. ARKIVOC 2001, (ii), 133. (d) Haider, N. J. Heterocyclic Chem. 2002, 39, 511. (e) Haider, N.; Sotelo, E. Chem. Pharm. Bull. 2002, 50, 1479. (f) Haider, N. Curr. Org. Chem. 2006, 10, 363 .

15. Haider, N.; Käferböck, J. Tetrahedron 2004, 60, 6495.

16. Benson, S. C.; Li, J.-H.; Snyder, J. K. J. Org. Chem. 1992, 57, 5285.

17. Moody, C. J. J. Chem. Soc., Perkin Trans. 1 1985, 2505.

18. Wanko, R. Ph.D. Thesis, University of Vienna, 1995.

19. Haider, N.; Wanko, R. Molecules 1999, 4, M105.

20. Plieninger, H.; Müller, W.; Weinerth, K. Chem. Ber. 1964, 97, 667.

21. For a review of the canthine alkaloids, cf. Ohmoto, T.; Koike, K. In The Alkaloids, Brossi, A., Ed., Academic Press: New York, 1989, Vol. 36, p 135.

22. Scudiero, D. A.; Shoemaker, R. H.; Paull, K. D.; Monks, A.; Tierney, S.; Nofziger, T. H.; Currens, M. J.; Seniff, D.; Boyd, M. R. Cancer Res. 1988, 48, 4827.

23. For some of the new compounds, microanalytical data indicated partial hydration. In all of these cases, residual water could not be removed even on prolonged drying in vacuo over $\mathrm{P}_{2} \mathrm{O}_{5}$. 